\section{Journal of Radiotherapy in} Practice

\section{cambridge.org/jrp}

\section{Original Article}

Cite this article: Olanloye EE, Ramlaul A, Ntekim Al, and Adeyemi SS. (2022) FDG-PET/CT and MR imaging for target volume delineation in rectal cancer radiotherapy treatment planning: a systematic review. Journal of Radiotherapy in Practice 21: 529-539. doi: $10.1017 /$ S1460396921000388

\section{Received: 19 March 2021}

Revised: 18 April 2021

Accepted: 21 April 2021

First published online: 8 September 2021

\section{Key words:}

FDG-PET/CT; MRI; radiotherapy treatment planning; rectal cancer; target volume delineation

\section{Author for correspondence:}

Elizabeth Olanloye, Department of Radiation Oncology, University College Hospital, Ibadan, Nigeria. Tel: 02348032713611.

E-mail: ebunoluwayinka@gmail.com

\section{FDG-PET/CT and MR imaging for target volume delineation in rectal cancer radiotherapy treatment planning: a systematic review}

Elizabeth E. Olanloye ${ }^{1,2}\left(\mathbb{0}\right.$, Aarthi Ramlaul $^{1}$, Atara I. Ntekim ${ }^{2}$ and Segun S. Adeyemi ${ }^{1}$

${ }^{1}$ Department of Allied Health Professions, Midwifery and Social Work, University of Hertfordshire, Hatfield, UK and ${ }^{2}$ Department of Radiation Oncology, College of Medicine, University of Ibadan/University College Hospital, Ibadan, Nigeria

\begin{abstract}
Aim: The aim of this systematic review was to synthesise and summarise evidence surrounding the clinical use of fluoro-2-deoxy-d-glucose positron emission tomography/computed tomography (FDG-PET/CT) and magnetic resonance imaging (MRI) for target volume delineation (TVD) in rectal cancer radiotherapy planning.

Methods: PubMed, EMBASE, Cochrane library, CINAHL, Web of Science and Scopus databases and other sources were systematically queried using keywords and relevant synonyms. Eligible full-text studies were assessed for methodological quality using the QUADAS-2 tool. Results: Eight of the 1448 studies identified met the inclusion criteria. Findings showed that MRI significantly delineate larger tumour volumes (TVs) than FDG-PET/CT while diffusion-weighted magnetic resonance imaging (DW-MRI) defined smaller gross tumour volumes (GTVs) compared to T2 weighted-Magnetic Resonance Image. CT-based GTVs were found to be larger compared to FDG-PET/CT. FDG-PET/CT also identified new lesions in 15-17\% patients and TVs extending outside the routinely used clinical standard CT TV in $29-83 \%$ patients. Between observers, delineated volumes were similar and consistent between MRI sequences, whereas interobserver agreement was significantly improved with FDG-PET/CT than CT.

Conclusion: FDG-PET/CT and DW-MRI appear to delineate smaller rectal TVs and show improved interobserver variability. Overall, this study provides valuable insights into the amount of attention in the research literature that has been paid to imaging for TVD in rectal cancer.
\end{abstract}

(c) The Author(s), 2021. Published by Cambridge University Press. This is an Open Access article, distributed under the terms of the Creative Commons Attribution-NonCommercialNoDerivatives licence (http:// creativecommons.org/licenses/by-nc-nd/4.0/), which permits non-commercial re-use, distribution, and reproduction in any medium, provided the original work is unaltered and is properly cited. The written permission of Cambridge University Press must be obtained for commercial re-use or in order to create a derivative work.

\section{CAMBRIDGE} UNIVERSITY PRESS

\section{Introduction}

Colorectal cancer (CRC) is the third most common cancer after lung cancer and breast cancer prevalent in both sexes worldwide with over 1.3 million new cases and 693,933 deaths estimated to have occurred in $2012^{1}$ compared to 1.8 million new cases and 881,000 deaths in $2018 .^{2}$ Data from western-industrialised countries and global cancer statistics have shown that approximately one-third of CRCs cases occur in the rectum. ${ }^{2-4}$ As the risk of local recurrence is a key issue in rectal cancer, a multidisciplinary approach involving surgery, radiotherapy and chemotherapy is the mainstay of the management process. ${ }^{5,6}$ Radiotherapy a non-invasive method, combined with chemotherapy, that is, preoperative chemoradiotherapy (PCRT) has been the standard treatment of choice for resectable and/or locally advanced rectal cancers (LARC). ${ }^{7,8}$ This approach has been reported to improve tumour downstaging which may allow tumour resectability, sphincter conservation ${ }^{7}$ or non-operative management. ${ }^{9}$ Several randomised trials have demonstrated improved 5-year locoregional control rates of $91 \cdot 9-94 \% .^{10,11}$

Radiotherapy generally aims to deliver prescribed radiation dose to target tumour volume (TV) while sparing adjacent normal surrounding tissues. Imaging in radiotherapy plays a crucial role for tumour localisation and target volume delineation (TVD) for radiotherapy treatment planning (RTP), verification of patient positioning for reproducibility and more recently for high dose delivery. ${ }^{12}$ Currently, the mainstay of radiotherapy delivery technique for rectal cancer has been based on 3D conformal radiotherapy and advanced conformal techniques, namely, intensity-modulated radiotherapy (IMRT), volumetric-modulated arc therapy and imageguided radiotherapy (IGRT). ${ }^{13}$ The advent of these techniques has demonstrated beneficial therapeutic ratio effects by maximising dose to the TV, reducing radiation toxicity to organs at risk (OAR), increasing the chance of local tumour control, and consequently, improving the quality of life. ${ }^{14}$ However, the success of these techniques requires excellent imaging quality for accurate visualisation and delineation of the TV and OAR. ${ }^{12,13}$ 
Lately, computed tomography (CT) imaging has been referred to as the gold standard for image-based conformal radiotherapy planning for all cancers. ${ }^{12,15}$ Despite its advantages, a number of studies have reported significant inter- and intra-observer variation and overestimation of TVs with CT planning owing to its inadequate soft tissue contrast. ${ }^{4,16}$

Recently, magnetic resonance imaging (MRI) is at the heart of rectal cancer staging and radiotherapy planning due to its superb soft tissue discrimination and multiplanar capabilities. ${ }^{17,18} \mathrm{MRI}$ in RTP is now well established to overcome the limitations of CT in rectal cancer RTP as it provides additional information that allows for detailed and accurate evaluation of the rectal wall and local tumour extent. ${ }^{13}$ This could consequently result in smaller but accurate tumour contours thereby enabling dose constraint to the OAR and tumour dose optimisation. ${ }^{19}$ MRI can provide functional information in addition to anatomical imaging. ${ }^{20}$ The past decade has seen the rapid development of MRI from a complimentary modality to CT due to its geometric distortion and lack of electron density ${ }^{18,21}$ to MRI-only simulation for radiotherapy planning. ${ }^{21,22}$ Studies have been carried out using modified imaging sequences and distortion correction techniques to eliminate image distortion. ${ }^{21}$ Also, dose calculation in MRI has been enabled using synthetic or pseudo-CT density data. ${ }^{23,24}$ More recently, integrated MR with teletherapy units, that is, Cobalt or Linear accelerator has been developed to provide real time IGRT for adaptive treatment techniques. ${ }^{20}$ However, this imaging technology has not escaped some limitations, ${ }^{20}$ and importantly, there remains a paucity of evidence on its application in RTP.

Currently, attention has also focused on the use of ${ }^{18}$ Fluorine-2fluoro-2-deoxy-D-glucose or fluoro-2-deoxy-d-glucose-positron emission tomography/CT (FDG-PET/CT) combining functional and anatomical imaging to provide more detailed information on tumour metabolic activity in RTP. ${ }^{25}$ In contrast to morphological information provided by anatomical-base modalities, that is, MRI and CT, the advantages of PET/CT include: automatic creation of a delineation around the tumour thereby providing smaller but more accurate TVs. This allows for tumour dose escalation with minimal dose to the OAR, reduced interobserver variability, with the potential to alter treatment strategy. ${ }^{13,16,26}$ This sometimes detects new lesions thus minimizing geographical miss. PET/CT has been shown to supersede MR and CT imaging in many cancer staging. ${ }^{27}$

The International Atomic Energy Agency (IAEA) suggests that RTP should always be based on the most accurate available assessment of tumour extent. ${ }^{28}$ In other words, the gold standard diagnostic modality for staging any cancer may be appropriate for its RTP. The only available systematic review which examined the benefits of MRI and PET over CT in TVD identified a paucity of evidence, indefinable optimal standard imaging sequence for MR rectal RTP and the methodological approaches with FDG-PET/CT TVD as major issues of concern. ${ }^{29}$ The need to undertake a current systematic review is imperative due to the limited databases searched and also to assess what has been added to the evidence base since the previous version of the review.

The aim of this systematic review was to synthesise available data to provide a summary of evidence and compare the role of FDG-PET/CT and MRI with conventional CT imaging in TVD of patients with rectal cancer with regard to optimal TV definition and extent of interobserver variation (IOV).

\section{Materials and Methods}

\section{Search strategy}

An extensive systematic literature search was conducted from January 2005 to December 2018 in six electronic databases including MEDLINE PubMed, EMBASE, Cochrane library, CINAHL, Web of Science and Scopus, using the search terms rectal cancer, radiotherapy planning, FDG-PET/CT, MRI and CT with relevant synonyms. Search terms were classified using the PICO (patients or target condition, index tests, comparator and outcome) concept. ${ }^{30}$ Commencing search date from 2005 was to ensure that publications that may have been missed in the previous review possibly due to delayed period in publication are included in this study. Details of the search strategies are presented in Appendix 1 (Appendix 1 available as supplementary material).

\section{Study selection}

This systematic review was conducted and reported in accordance with the Preferred Reporting Items for Systematic Reviews and Meta-Analysis (PRISMA) statement guidelines. ${ }^{31}$

Studies were initially selected independently by one of the authors (EO) based on predefined eligibility criteria and then subsequently by one of the other authors (AN). The initial stage reviewed titles and abstracts of studies to select suitable articles and removed duplicates. Subsequent review focused on full text to identify eligible studies. ${ }^{31}$ To be eligible, studies must include primary publications such as randomised studies, comparative observational studies or studies on CT, MR and PET/CT imaging for TVD for RTP of rectal cancer involving external radiotherapy treatment. Abstracts were excluded from the review in order to reduce publication bias partly due to high variability in their reliability as the full studies were not published. ${ }^{30,31}$ The list with the exclusion criteria data is provided in Appendix 2 (Appendix 2 available as supplementary material).

\section{Data extraction}

A custom designed electronic data extraction form based on Cochrane collaboration ${ }^{30}$ was developed, pilot-tested on four included studies to identify any missing or surplus data and then refined accordingly. Corresponding study authors were contacted in case of missing or unclear and additional information such as the release of individual patient data. Data were primarily extracted by one of the authors (EO) and subsequently checked with two other authors. The extracted data included first author, year of publication, methods of study, participants characteristics, interventions and comparison (MRI, FDG-PET/CT and CT), and outcome measures of interest.

\section{Quality assessment}

The Quality Assessment of Diagnostic Accuracy Studies (QUADAS-2) ${ }^{32}$ tool, a domain-based approach was used to assess the quality of included studies in this review. The quality assessment addressed risk of bias (internal validity or methodological quality) and concerns about applicability (external validity or directness) for each eligible study. Signalling questions in each of the four domains were rated with answer options low, high or unclear. 


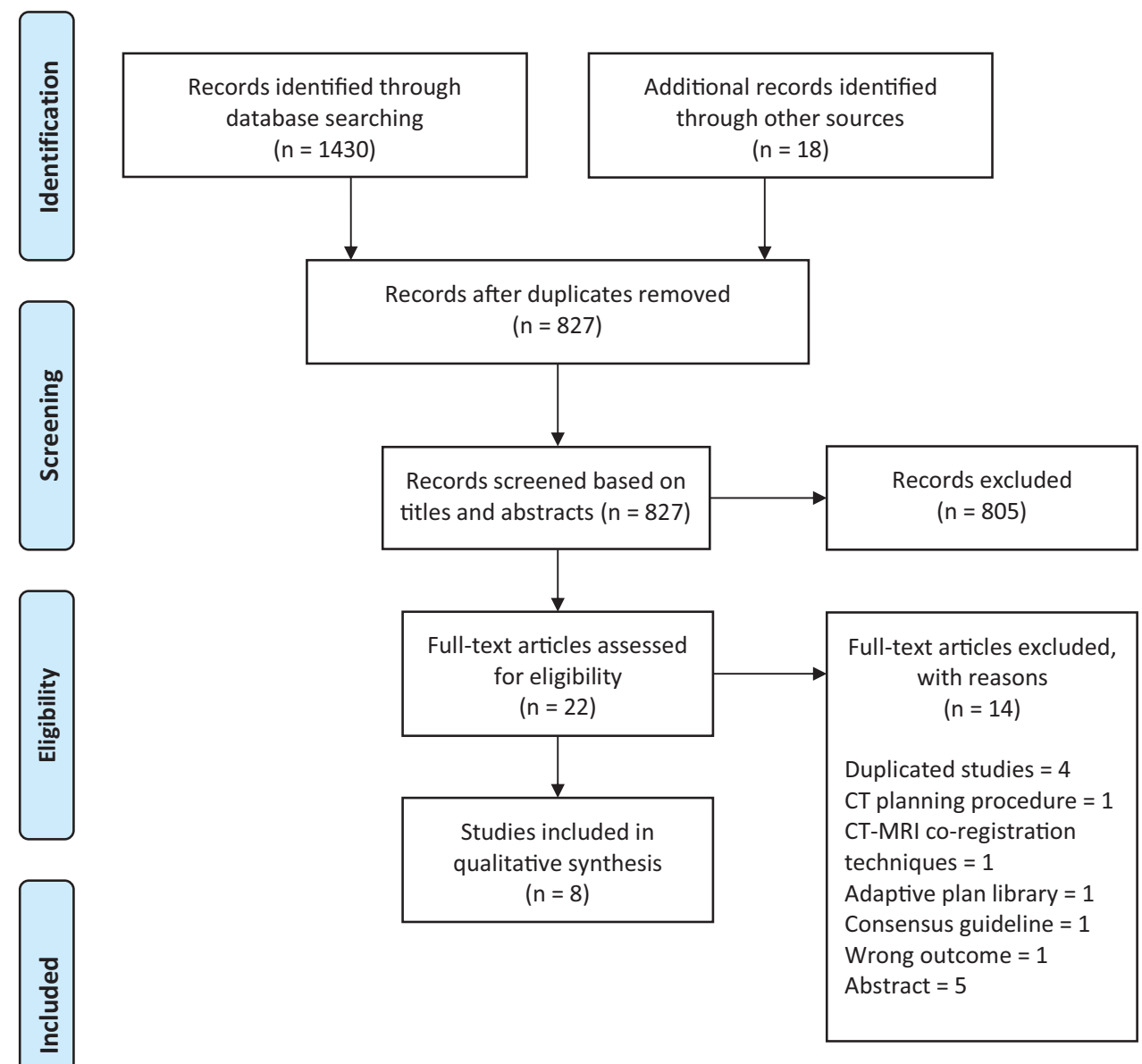

Figure 1. PRISMA flow diagram summarising the study selection process.

\section{Data analysis}

Extracted data were processed where necessary before presentation in tables. A narrative synthesis approach was adopted to summarise and synthesise findings from all included studies. The primary outcome measured was the summary statistics (means) for FDG-PET/CT, MRI and CT delineated TVs while the secondary outcome was interobserver variability.

\section{Results}

\section{Study selection}

A total of eight studies were identified for inclusion in the review. The literature search identified a total of 1448 studies. After removing duplication, 827 studies remained. Of these, 805 studies were excluded based on the eligibility criteria after reading their titles and abstract. Another nine studies were discarded after detailed screening of the full text. Five additional studies were excluded as full text of the study was unavailable. No studies were identified by checking the reference list neither were unpublished relevant studies available. A PRISMA flow diagram summarising the selection process is presented in Figure 1.

\section{Study characteristics}

Only one ${ }^{33}$ out of the eight studies selected for the review mentioned the study design used. ${ }^{34-40}$ Two of the included studies were conducted using prospective design. The included studies involved 261 patients with LARC. All patients were planned for PCRT with majority $(87 \cdot 5 \%)$ to undergo long-course preoperative chemoradiotherapy. Of the six studies that provided detailed participants characteristics, 83/199 were females, with ages between 26 and 89 years. Only one of the eight included studies was multicentre while others were single-centre studies. The characteristics of the included studies are summarised in Table 1. Data for the outcome variables, TV and IOV, are provided in Tables 2-4. A meta-analysis though initially planned was not feasible due to heterogeneity across and between the included studies.

\section{Risk of bias within studies}

The quality assessment with regards to risk of bias within individual studies were judged 'low risk of bias' in three studies and 'at risk of bias' in five studies as three studies were judged unclear in one domain. ${ }^{35,38,39}$ One study ${ }^{33}$ was judged high risk in one domain, while Kilic et al. ${ }^{40}$ was judged unclear in three domains. In terms of applicability, all included studies had an overall judgement of 'low concern' (see Figure 2).

\section{Narrative synthesis}

Relating to the TV outcome, all studies included in the review were classified into three different groups based on the imaging tests (see Tables 2 and 3). Two studies compared MR with PET/CT imaging including a total of 83 patients. ${ }^{34,38}$ Their results 
Table 1. Characteristics of studies included in the review

\begin{tabular}{|c|c|c|c|c|c|c|c|c|c|}
\hline Author, year, country & $\begin{array}{l}\text { Study design, patient } \\
\text { selection }\end{array}$ & Study period (years) & $\mathrm{N}$ & $\begin{array}{l}\text { Mean age } \\
\text { (range) }\end{array}$ & $\begin{array}{l}\text { Fem } \\
(\%)\end{array}$ & Disease stage & $\begin{array}{l}\text { Radiotherapy- } \\
\text { strategy, technique }\end{array}$ & $\begin{array}{l}\text { Imaging modality, time } \\
\text { interval }^{\mathrm{a}}\end{array}$ & $\begin{array}{l}\text { Outcome } \\
\text { measured }\end{array}$ \\
\hline $\begin{array}{l}\text { bBraendengen, 2011, } \\
\text { Sweden }^{38}\end{array}$ & $\begin{array}{l}\text { Cross-sectional } \\
\text { prospective design, } \\
\text { consecutive patients }\end{array}$ & $\begin{array}{l}\text { November 2007- } \\
\text { December } 2009\end{array}$ & 68 & $\begin{array}{l}66 \text { (median) } \\
\quad(33-87)\end{array}$ & 60 & $\begin{array}{l}\text { LARC (cT4a, } \\
\left.\text { cT3+ mrf }+ \text { or }^{\star}\right)\end{array}$ & LCPCRT, NR & $\begin{array}{l}\text { MRI Vs FDG-PET/CT, median } \\
\text { interval }=30 \text { days } \\
\text { (maximum }=58 \text { days) }\end{array}$ & GTV \\
\hline $\begin{array}{l}\text { Buijsen, 2012, } \\
\text { Netherlands }{ }^{35}\end{array}$ & NR & NR & 42 & NR & NR & CT2-4N0-2M0 & LCPCRT, NR & $\begin{array}{l}\text { FDG-PET/CT Vs CT, } \\
\text { consecutive scans on the } \\
\text { same day }\end{array}$ & GTV, CTV, IV \\
\hline $\begin{array}{l}\text { Burbach, 2016, } \\
\text { Netherland }{ }^{39}\end{array}$ & $\begin{array}{l}\text { Cross-sectional } \\
\text { prospective design, } \\
\text { consecutive patients }\end{array}$ & NR & 24 & $\begin{array}{l}66 \text { (median) } \\
(26-80)\end{array}$ & 17 & $\begin{array}{l}\text { LARC (T3 with } \\
\text { threatened mrf, } \\
\text { T4 or N2M0) }\end{array}$ & ?PCRT, NR & $\begin{array}{l}\text { MRI: T2W Vs DWI-SPLICE Vs } \\
\text { combi, consecutive scans } \\
\text { on the same day }\end{array}$ & $\begin{array}{l}\text { GTV, } \\
\text { interobserver } \\
\text { variation (IV) }\end{array}$ \\
\hline Kilic, 2015, Turkey ${ }^{40}$ & $\begin{array}{l}\text { Cross-sectional } \\
\text { retrospective design, } \\
\text { NR }\end{array}$ & $\begin{array}{l}\text { March 2008- } \\
\text { July } 2011\end{array}$ & 20 & NR & NR & LARC & $\begin{array}{l}\text { LCPCRT, 3D-CRT Vs } \\
\text { IMRT }\end{array}$ & FDG-PET/CT Vs CT, NR & CTV \\
\hline Regini, 2014, UK ${ }^{36}$ & $\begin{array}{l}\text { Cross-sectional } \\
\text { retrospective design, } \\
\text { consecutive patients }\end{array}$ & $\begin{array}{l}\text { January 2008- } \\
\text { January } 2012\end{array}$ & 27 & $66.9(27 \cdot 1-88 \cdot 8)$ & 44 & LARC & LCPCRT, 3D-CRT & $\begin{array}{l}\text { MRI: T2W Vs DWI, } \\
\text { consecutive scans on the } \\
\text { same day }\end{array}$ & GTV, IV \\
\hline Roels, 2009, Belgium ${ }^{34}$ & NR & $\begin{array}{l}\text { May 2005-August } \\
2007\end{array}$ & 15 & **54.5 (48-82) & 27 & T2/3-N1/2MO & LCPCRT, 3D-CRT & $\begin{array}{l}\text { MRI Vs FDG-PET/CT, MRI } \\
\text { performed within } 24 \text { hours } \\
\text { from FDG-PET/CT }\end{array}$ & TV \\
\hline Whaley, 2014, USA ${ }^{37}$ & $\begin{array}{l}\text { Cross-sectional } \\
\text { retrospective design, } \\
\text { consecutive patients }\end{array}$ & $\begin{array}{l}\text { January 2009- } \\
\text { June } 2011\end{array}$ & 34 & $56(33-73)$ & 24 & T3-4NO-2MO-1 & LCPCRT, NR & $\begin{array}{l}\mathrm{PET} / \mathrm{CT} \text { Vs } \mathrm{CT} \text {, consecutive } \\
\text { scans on the same day }\end{array}$ & GTV, IV \\
\hline Withofs, 2014, Belgium 33 & $\begin{array}{l}\text { Cross-sectional } \\
\text { retrospective design, } \\
\text { consecutive patients }\end{array}$ & $\begin{array}{l}\text { April 2009- } \\
\text { June } 2010\end{array}$ & 31 & $65(29-82)$ & 45 & LARC & LCPCRT, NR & $\begin{array}{l}\text { FDG-PET/CT Vs CT, median } \\
\text { (range) delay }=5 \text { (1- } \\
12 \text { days) }\end{array}$ & $\begin{array}{l}\text { GTV Vs MTVs, } \\
\text { IV }\end{array}$ \\
\hline
\end{tabular}

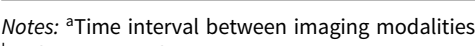

Multicentre study.

or ${ }^{*}$, or with radiologically malignant lateral lymph nodes outside the mesorectum.

54.5 , combined groups calculation.

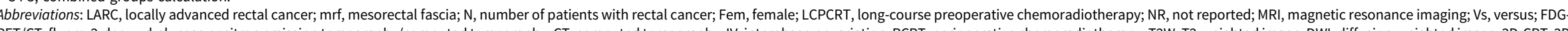

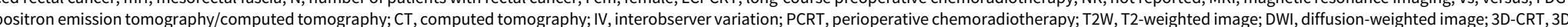

conformal radiotherapy; IMRT, intensity-modulated radiotherapy; MTVs, metabolic tumour volumes; GTV, gross tumour volume; CTV, clinical target volume; TV, tumour volume; combi, combination. 
Table 2. Outcome variable: MRI studies on TVD

\begin{tabular}{|c|c|c|c|c|c|}
\hline Author & Participants & Index test & Comparator & Outcome measures & Results \\
\hline Braendengen $^{38}$ & 68 & $\begin{array}{l}\mathrm{PET} / \mathrm{CT} \text { : Scans performed with a Siemens } \\
\text { Biograph } 64 \text { ( } 45 \text { patients), a GE Discovery } \\
\text { STE (20 patients) and a GE Discovery ST ( } 3 \\
\text { patients). }{ }^{18} \\
\text { F-FDG tracer used. Images acquired were } \\
\text { transverse, sagittal and coronal reformation } \\
\text { of the PET, CT and fused PET/CT images } \\
\text { with slice thickness of } 3 \cdot 75-5 \mathrm{~mm} \text {. Patients } \\
\text { were scanned in supine position and in the } \\
\text { radiotherapy treatment position }\end{array}$ & $\begin{array}{l}1.5 \text { T MRI system. Patients scanned in } \\
\text { supine position. Scan protocols } \\
\text { included axial and sagittal T2W-fast } \\
\text { spin-echo and T1-WI of the pelvic area. } \\
\text { Slice thicknesses were between } \\
3-5 \mathrm{~mm} \text {. Forty-one patients were } \\
\text { scanned at the referring hospital and } \\
27 \text { patients were scanned at another } \\
\text { study centre }\end{array}$ & $\begin{array}{l}\text { GTV as delineated on MRI and FDG- } \\
\text { PET/CT. Both GTV volumes were } \\
\text { manually delineated by two physicians } \\
\text { with specialists in clinical oncology and } \\
\text { radiotherapy, and one radiologist }\end{array}$ & $\begin{array}{l}\text { GTV-MRI volumes were larger than PET/ } \\
\text { CT volumes: mean } \pm \text { SD } 153 \pm 125 \mathrm{~cm}^{3} \\
\text { Vs. } 121 \pm 101 \mathrm{~cm}^{3}(p<0 \cdot 001) \text {. Union of } \\
\text { MRI-PET-GTVs increased with a mean of } \\
10 \cdot 5 \% \text { above the standard GTV-MRI. } \\
\text { With PET/CT, } 15 \% \text { potentially had } \\
\text { change in treatment plan due to new } \\
\text { lesion seen }\end{array}$ \\
\hline Roels $^{34}$ & 15 & $\begin{array}{l}\text { PET/CT: using Siemens Biograph } 2 \text { scanner } \\
\text { (4 patients) or Siemens HiRez Biograph } \\
\text { scanner ( } 11 \text { patients). }{ }^{18} \text { F-FDG tracer } \\
\text { injected. Examination included a contrast- } \\
\text { enhanced, high dose spiral CT scan } \\
\text { followed by a PET scan in the caudocranial } \\
\text { direction. PET/CT scans performed with } \\
\text { patients prone in treatment position. PET } \\
\text { data reconstructed using maximisation } \\
\text { algorithm and attenuation correction from } \\
\text { CT data }\end{array}$ & $\begin{array}{l}\text { 1.5-T MRI system with dedicated } \\
\text { surface coil was used to simulate the } \\
\text { patients within } 24 \text { hours after FDG- } \\
\text { PET/CT. Patients were simulated in } \\
\text { supine position on the MRI table. } \\
\text { Rectal contrast was utilised. T2W turbo } \\
\text { spin-echo images were acquired in } \\
\text { sagittal, transaxial and coronal } \\
\text { sections. T1W-images in axial sections } \\
\text { were also acquired }\end{array}$ & $\begin{array}{l}\text { TVs delineated on the images before } \\
\text { treatment. MRI was independently } \\
\text { delineated by an experienced } \\
\text { radiologist. FDG-PET volumes were } \\
\text { automatically segmented using SBR } \\
\text { and GR methods }\end{array}$ & $\begin{array}{l}\text { The mean MRI-TV was larger } \\
27 \cdot 10 \pm 16 \cdot 50 \mathrm{~cm}^{3} \text { when compared to } \\
\text { FDG-PET TV (SBR) } 18 \cdot 30 \pm 12 \cdot 40 \mathrm{~cm}^{3} \text { and } \\
\text { FDG-PET TV (GR) } 15.50 \pm 10 \cdot 60 \mathrm{~cm}^{3} \\
(p<0.001)\end{array}$ \\
\hline Burbach $^{39}$ & 24 & $\begin{array}{l}\text { MRI scans were performed on two (Philips } \\
\text { Ingenia Wide-Bore or Achieva } 3 \text { TX) } 3 \text { T MRI } \\
\text { scanners. No bowel preparation prior } \\
\text { scanning. Patients were scanned supine on } \\
\text { a flat table-top. No contrast was } \\
\text { administered. DWI-SPLICE }\left(b=800 \mathrm{~s} / \mathrm{mm}^{2}\right) \\
\text { images were acquired in axial planes with } \\
\text { resolution: } 2.02-2.5 \times 2.02-2.5 \mathrm{~mm} \text { and slice } \\
\text { thickness: } 4.0 \mathrm{~mm}\end{array}$ & $\begin{array}{l}\text { MRI scans were performed on two } \\
\text { (Philips Ingenia Wide-Bore or Achieva } \\
\text { 3TX) } 3 \text { T MRI scanners. No bowel } \\
\text { preparation prior scanning. Patients } \\
\text { were scanned supine on a flat table- } \\
\text { top. No contrast was administered. } \\
\text { T2W-images were acquired in } \\
\text { transverse planes with resolution: } \\
0.8-0.9 \times 0.85-1.07 \mathrm{~mm} \text { and slice } \\
\text { thickness: } 3.0 \mathrm{~mm}\end{array}$ & $\begin{array}{l}\text { GTV as identified on the images. Three } \\
\text { independent observers (1 radiation } \\
\text { oncologist with } 10 \text {-years-experience, } 1 \\
\text { radiation oncologist in-training with } 5 \text { - } \\
\text { years-experience and } 1 \text { researcher with } \\
\text { 2-years-experience) blindly delineated } \\
\text { from each other }\end{array}$ & $\begin{array}{l}\text { No significant volume differences } \\
\text { between observers per modality. T2W } \\
\text { volumes were significantly larger than } \\
\text { DWI and combi. The mean GTV volumes } \\
\text { for T2W, DWI and combi were } \\
63 \cdot 16 \pm 33 \cdot 39,41 \cdot 94 \pm 25 \cdot 49 \text { and } \\
54 \cdot 63 \pm 29 \cdot 54 \text {, respectively }\end{array}$ \\
\hline $\operatorname{Regini}^{36}$ & 27 & $\begin{array}{l}\text { 1.5 T MRI scanner using phased array body } \\
\text { coils. Patients were scanned caudo-cranially } \\
\text { in a supine position. A single shot SE-EPI } \\
\text { axial DWI (TR/TE: } 3000 / 77 \mathrm{~ms} \text { ) was acquired } \\
\text { in addition to T2W and T1W images }\end{array}$ & $\begin{array}{l}1.5 \text { T MRI scanner using phased array } \\
\text { body coils. Patients were scanned } \\
\text { caudo-cranially in a supine position. } \\
\text { Imaging sequences acquired included } \\
\text { T2W sagittal } \\
\text { (TR/TE: } 5280 / 97 \mathrm{~ms}) \text {, axial T1W SE (TR/ } \\
\text { TE: } 570 / 12 \mathrm{~ms} \text { ), axial T2W TSE (TR/TE: } \\
6600 / 90 \mathrm{~ms}) \text {, high resolution axial } \\
\text { oblique and coronal T2W TSE } \\
\text { (TR/TE: } 6600 / 90 \mathrm{~ms}) \text {. No contrast agent } \\
\text { administered. No patient preparation } \\
\text { before examination }\end{array}$ & $\begin{array}{l}\text { GTVs delineated on both imaging tests. } \\
\text { These were independently delineated } \\
\text { by two experienced radiologists }\end{array}$ & $\begin{array}{l}\text { The median T2W volumes were } \\
\text { marginally larger but statistically } \\
\text { non-significant from DWI volumes: } \\
38.88 \mathrm{~cm}^{3} \text { and } 36.92 \mathrm{~cm}^{3} \text {, respectively }\end{array}$ \\
\hline
\end{tabular}


Table 3. Outcome variable: PET/CT versus $C T$ studies on TVD

\begin{tabular}{|c|c|c|c|c|c|}
\hline Author & Participants & Index test & Comparator & Outcome measures & Results \\
\hline Buijsen 35 & 42 & $\begin{array}{l}\text { Integrated PET/CT scanner (Truepoint } \\
\text { Biograph } 40 \text {, Siemens) was used. Patients } \\
\text { fasted for at least } 6 \text { hours prior } \\
\text { intravenous FDG tracer injection was } \\
\text { administered. Patients were positioned } \\
\text { on a flat table top using mobile laser } \\
\text { alignment in a head-first supine position. } \\
\text { Scans were performed in sequence: } \\
\text { a topogram, followed by a CT scan, FDG } \\
\text { tracer was injected before the PET scan. } \\
\text { PET images were reconstructed from the } \\
\text { acquired list mode data }{ }^{41}\end{array}$ & $\begin{array}{l}\text { CT scan conducted in the same patient } \\
\text { position set-up }\end{array}$ & $\begin{array}{l}\text { GTV and CTV as delineated on CT and } \\
\text { fused PET/CT images. GTV volumes were } \\
\text { delineated independently and blindly by } 2 \\
\text { radiation-oncologists, } 1 \text { senior resident, } 1 \\
\text { radiation-technologist and } 1 \text { radiologist in } \\
\text { three rounds. Round } 1 \text { : GTV-CT, round } 2 \text { : } \\
\text { GTV-PET/CT-manual, round 3: editing the } \\
\text { automatic delineation generated on the } \\
\text { GTV-PET/CT-auto-SBR. A minimum interval } \\
\text { between each round was } 4 \text { weeks. CTV } \\
\text { volumes were delineated by three } \\
\text { observers. }\end{array}$ & $\begin{array}{l}\text { The GTV volumes were significantly larger } \\
\text { using CT than PET/CT. The mean PET/CT- } \\
\text { auto, PET/CT-manual, CT and edited } \\
\text { PET/CT-auto volumes were } 23 \cdot 6 \mathrm{~cm}^{3} \text {, } \\
28.8 \mathrm{~cm}^{3}, 46 \cdot 8 \mathrm{~cm}^{3} \text { and } 18 \cdot 2 \mathrm{~cm}^{3} \\
(p<0.001) \text {. The CTV based on PET } \\
\text { extended outside the complete CTV used } \\
\text { in daily clinical practice in about } 29 \% \\
\text { of patients }\end{array}$ \\
\hline Kilic ${ }^{40}$ & 20 & NR & NR & $\begin{array}{l}\text { GTV and CTV as delineated on CT and } \\
\text { PET/CT. These volumes were manually } \\
\text { delineated by an experienced radiation } \\
\text { oncologist and verified by a second } \\
\text { independent physician }\end{array}$ & $\begin{array}{l}\text { The CTV-PET/CT volumes were larger than } \\
\text { that of CT: mean values were } \\
51.74 \pm 14.05 \mathrm{~cm}^{3} \mathrm{Vs} 46.26 \pm 12.91 \mathrm{~cm}^{3} \\
(p<0.043)\end{array}$ \\
\hline Whaley ${ }^{37}$ & 30 & $\begin{array}{l}\text { Integrated Philips Gemini TF Big Bore } \\
\text { PET/CT scanner system. Patients fasted } \\
8 \text { hours examination. Patients' blood } \\
\text { glucose level was assessed prior FDG } \\
\text { tracer was injected. First, CT simulation } \\
\text { was conducted followed by the PET scan. } \\
\text { Both scans were acquired with patients } \\
\text { in the same position as with radiotherapy } \\
\text { treatment. Knee-foot lock was applied for } \\
\text { immobilisation. PET data were } \\
\text { reconstructed using the CT images for } \\
\text { attenuation correction }\end{array}$ & $\begin{array}{l}\text { CT simulation performed using the same } \\
\text { scanner and patient position set-up as } \\
\text { the PET scan. Slice thickness: } 3 \mathrm{~mm}\end{array}$ & $\begin{array}{l}\text { GTVs identified on the imaging studies. } \\
\text { Three radiation oncologists delineated the } \\
\text { volumes independently and blinded from } \\
\text { each other. A minimum interval of } 2 \text { weeks } \\
\text { waiting period was observed between the } \\
\text { two-delineation process }\end{array}$ & $\begin{array}{l}\text { The mean PET/CT and CT volumes were } \\
88 \cdot 1 \mathrm{~cm}^{3} \text { and } 102 \cdot 8 \mathrm{~cm}^{3} \text {, respectively } \\
(p<0.03) \cdot \mathrm{GTV}-\mathrm{PET} / \mathrm{CT} \text { extended outside } \\
\text { the standard GTV-CT in } 83 \% \text { of patients. } \\
17 \% \text { ( } 5 \text { patients) had changes in } \\
\text { treatment planning }\end{array}$ \\
\hline Withofs ${ }^{33}$ & 31 & $\begin{array}{l}\text { PET/CT studies were conducted on a } \\
\text { Gemini TF PET/CT system. Patients were } \\
\text { positioned supine in the treatment } \\
\text { position on a flat pallet using a dedicated } \\
\text { laser system. A low dose CT scan with } \\
5 \mathrm{~mm} \text { slice thickness was followed by the } \\
\text { PET scan. Data used for reconstruction } \\
\text { included time of flight, correction for } \\
\text { decay, scatter, random and CT-based } \\
\text { attenuation correction. Reconstructed } \\
\text { matrixes for CT and PET were: } 512 \times 512 \\
\text { and } 144 \times 144 \text {, respectively. Both PET and } \\
\text { CT data were co-registered }\end{array}$ & $\begin{array}{l}\text { A big Bore CT scanner was used to } \\
\text { perform CT-simulation for the patients. } \\
\text { Matrix used for } 21 / 31 \text { patients was } \\
1024 \times 1024 \text { while } 512 \times 512 \text { matrix size } \\
\text { was used for } 10 \text { patients. The low dose } \\
\text { CT and CT simulation data were } \\
\text { automatically co-registered }\end{array}$ & $\begin{array}{l}\text { GTV as delineated on CT-simulation and } \\
\text { MTVs as delineated on PET/CT. The GTV } \\
\text { was independently delineated by an } \\
\text { experienced radiation oncologist. The } \\
\text { MTVs were automatically delineated using } \\
\text { six different segmentation algorithms with } \\
\text { manual manipulation performed by an } \\
\text { experienced nuclear medicine physician }\end{array}$ & $\begin{array}{l}\text { All MTV values were significantly different } \\
\text { between the various methods } \\
(p<0.0001) \text {. The GTV-CT volume was } \\
\text { significantly larger than all MTVs. The } \\
\text { mean volumes included: GTV- } \\
\mathrm{CT}=40 \cdot 6 \pm 31 \cdot 28 \mathrm{~cm}^{3} \text { Vs MTVs } \\
\left(\mathrm{FLAB}=21 \cdot 36 \pm 16 \cdot 34 \mathrm{~cm}^{3} \text {, }\right. \\
\mathrm{GR}=18.97 \pm 16 \cdot 83 \mathrm{~cm}^{3} \text {, OASIS } \\
45 \%=15 \cdot 89 \pm 12.68 \mathrm{~cm}^{3} \text {, Philips } \\
45 \%=14.52 \pm 10 \cdot 91 \mathrm{~cm}^{3} \text {, OASIS } \\
2 \cdot 5=41 \cdot 62 \pm 33 \cdot 26 \mathrm{~cm}^{3} \text {, Philips } \\
\left.2.5=40 \pm 31.27 \mathrm{~cm}^{3}\right) \text {, respectively. }\end{array}$ \\
\hline
\end{tabular}

Abbreviations: refer to Table 1. 
Table 4. Outcome variable: studies on IOV

\begin{tabular}{|c|c|c|c|c|c|c|}
\hline Author & $\mathrm{N}$ & Imaging modality & Outcome measured & Image delineation & Method & Results \\
\hline \multirow[t]{2}{*}{ Burbach $^{39}$} & \multirow[t]{2}{*}{24} & \multirow{2}{*}{$\begin{array}{l}\text { MRI: T2W Vs DWI- } \\
\text { SPLICE Vs combi }\end{array}$} & \multirow{2}{*}{$\begin{array}{l}\text { Interobserver } \\
\text { variation between } \\
\text { the three } \\
\text { delineation } \\
\text { methods }\end{array}$} & 2 Radiation oncologists & \multirow{2}{*}{$\begin{array}{l}\text { Interobserver variation was examined by } \\
\text { calculating for conformity index (CI) per patient } \\
\text { per modality. A Fleiss' kappa was also } \\
\text { calculated to aid comparison with other studies }\end{array}$} & \multirow{2}{*}{$\begin{array}{l}\text { The mean } \mathrm{Cl} \text { for T2W, DWI and combi were } 0.70,0.71 \\
\text { and } 0.69 \text {, respectively, }(p>0.61)\end{array}$} \\
\hline & & & & 1 Researcher & & \\
\hline Regini $^{36}$ & 27 & MRI: T2W Vs DWI & $\begin{array}{l}\text { Interobserver } \\
\text { agreement in } \\
\text { volumes between } \\
\text { the two delineation } \\
\text { methods }\end{array}$ & 2 Radiologists & $\begin{array}{l}\text { Interobserver agreement was estimated using } \\
\text { Bland-Altman analysis: calculating mean } \\
\text { difference, } 95 \% \text { limits of agreement }\end{array}$ & $\begin{array}{l}\text { Interobserver agreement was not improved by DWI. The } \\
95 \% \text { limits of agreement were slightly wider for T2W } \\
\text { volumes than that of DWI. The mean difference (95\% } \\
\text { limits of agreement) were }-9.84(-54.96 \text { to }+35.28) \\
\mathrm{cm}^{3} \text { and }-14.79(-54.01 \text { to }+24.43) \mathrm{cm}^{3} \text { for T2W and } \\
\text { DWI volumes, respectively }\end{array}$ \\
\hline \multirow[t]{4}{*}{ Buijsen ${ }^{35}$} & \multirow[t]{4}{*}{42} & \multirow[t]{4}{*}{ FDG-PET/CT Vs CT } & \multirow{4}{*}{$\begin{array}{l}\text { Interobserver } \\
\text { variation between } \\
\text { delineated GTV } \\
\text { volumes }\end{array}$} & 2 radiation-oncologists, & \multirow{4}{*}{$\begin{array}{l}\text { GTV contours from the } 3 \text { different delineation } \\
\text { methods were compared by calculating the } \\
\text { concordance index }\end{array}$} & \multirow{4}{*}{$\begin{array}{l}\mathrm{PET} / \mathrm{CT} \text { significantly increased Cls with the best } \\
\text { interobserver agreement seen in PET/CT auto. The } \\
\text { mean } \mathrm{Cl} \text { for the } 5 \text { observers were: } 0.79 \pm 0.17 \text { using CT } \\
\text { alone in combination with MRI, } 0.82 \pm 0.16 \text { using PET/CT } \\
\text { manual and } 0.93 \pm 10.05(p<0.001) \text { using PET/CT } \\
\text { auto-contour }\end{array}$} \\
\hline & & & & 1 senior resident & & \\
\hline & & & & 1 radiation-technologist & & \\
\hline & & & & 1 radiologist & & \\
\hline Whaley ${ }^{37}$ & 30 & FDG-PET/CT Vs CT & $\begin{array}{l}\text { Interobserver } \\
\text { variability between } \\
\mathrm{CT} \text { and PET/CT GTV } \\
\text { volumes }\end{array}$ & 3 Radiation oncologists & $\begin{array}{l}\text { The interobserver concordance index }(\mathrm{CCl}) \text { was } \\
\text { calculated and compared using Wilcoxon } \\
\text { rank test }\end{array}$ & $\begin{array}{l}\text { PET/CT significantly increased interobserver CCI in GTV } \\
\text { delineation compared to CT delineation: } 0.56 \mathrm{Vs} 0.38 \\
(p<0.001)\end{array}$ \\
\hline \multirow[t]{2}{*}{ Withofs ${ }^{33}$} & \multirow[t]{2}{*}{31} & \multirow[t]{2}{*}{ FDG-PET/CT Vs CT } & \multirow{2}{*}{$\begin{array}{l}\text { Concordance } \\
\text { between all } \\
\text { volumes measured }\end{array}$} & $\begin{array}{l}1 \text { Radiation oncologist } \\
\text { delineated the GTV }\end{array}$ & \multirow{2}{*}{$\begin{array}{l}\text { The spatial distribution (sizes and shapes) of all } \\
\text { volumes were assessed for concordance by } \\
\text { comparing each MTV two by two and between } \\
\text { each MTV and GTV-CT using the conformity } \\
\text { index (CI) }\end{array}$} & \multirow{2}{*}{$\begin{array}{l}\text { Conformity indices differs significantly between the } \\
\text { various MTVs and GTV volumes. The mean } \mathrm{Cl} \text { between } \\
\text { the various MTVs and the GTV was }<0.4\end{array}$} \\
\hline & & & & $\begin{array}{l}\text { MTVs were automatically } \\
\text { delineated using six different } \\
\text { segmentation algorithms }\end{array}$ & & \\
\hline
\end{tabular}

Abbreviations: refer to Table 1. 


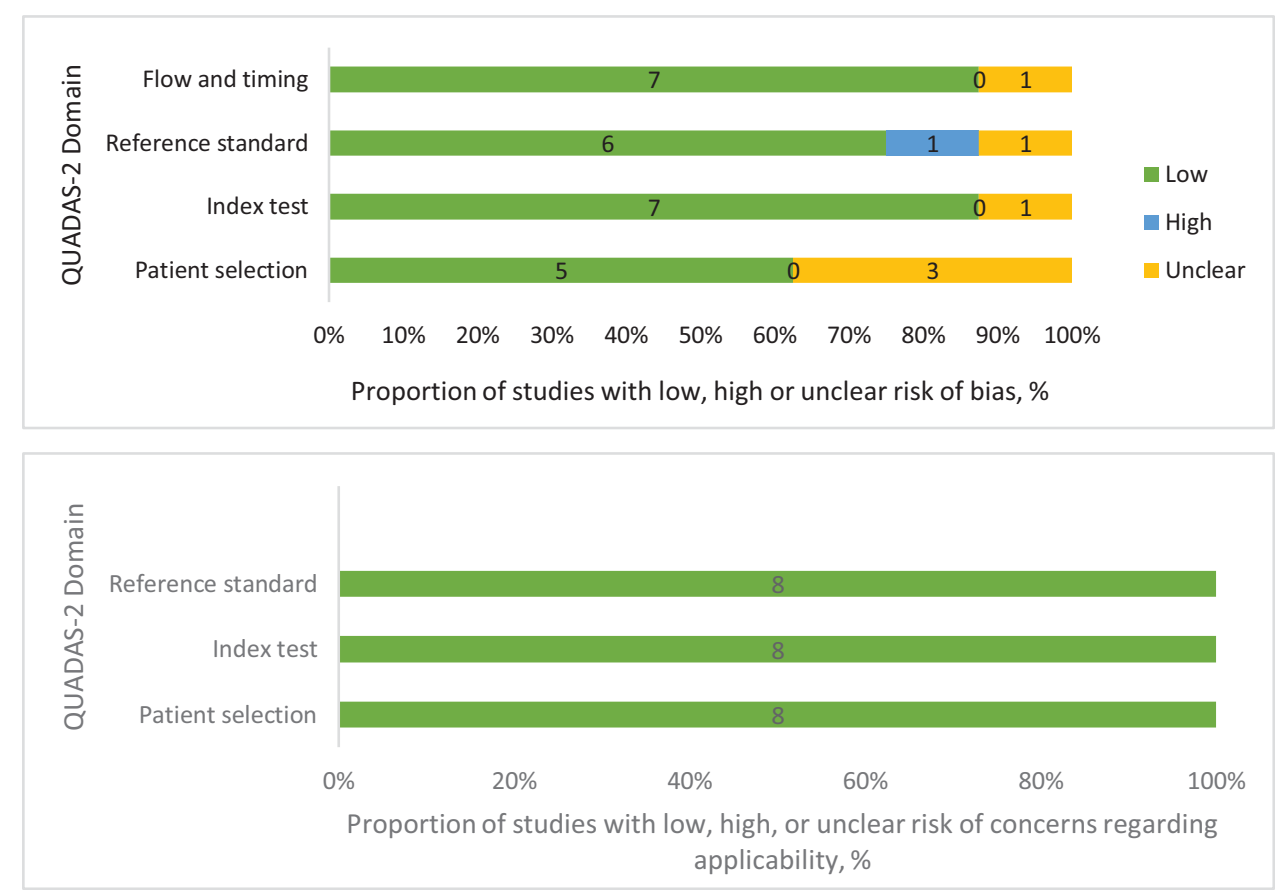

Figure 2. Recommended graphical display for QUADAS-2 results of risk of bias and concern regarding applicability of the included studies.

revealed that MRI significantly delineate larger TVs than PET/CT. PET/CT was also reported to provide additional information to standard delineation method as new lesions were identified in $15 \%$ of patients. ${ }^{38}$ The same authors in an attempt to co-register MR with PET/CT gross tumour volume (GTV), found that the volume increased with a median of $11 \%$ above the standard GTV-MRI.

A comparison between two MRI sequences, T2W and Diffusion Weighted Imaging (DWI), was reported in two studies which included 51 patients (Table 2). Although both studies found that DWI delineated smaller GTV volumes when compared with T2W, only one study found a statistically significant difference in volumes between the two approaches. ${ }^{39}$ The same study further showed that DWI consistently and significantly delineated the smallest volumes compared to co-registered T2W-DW and T2W sequences. The mean differences were: T2W versus $\mathrm{DWI}=19.85 \mathrm{~mL}(p<0.0001) ; \mathrm{T} 2 \mathrm{~W}$ versus $\mathrm{T} 2 \mathrm{~W}-\mathrm{DWI}=7 \cdot 16$ $\mathrm{mL} \quad(p<0.0001)$ and DWI versus T2W-DWI $=12.69 \mathrm{~mL}$ $(p<0.0001)$.

Four papers reported on PET/CT and CT in TVD (Table 3). Three of these articles found that GTV volumes delineated with CT were significantly larger; mean volume varied from $40.6 \mathrm{~cm}^{3}$ to $102 \cdot 8 \mathrm{~cm}^{3}$ compared with PET/CT delineated volumes. ${ }^{33,35,37}$ PET/CT based TVs extended outside the routinely used clinical standard CT-TVs in about $29-83 \%$ of patients, as shown by two of those papers. ${ }^{35,37}$ Whaley et al. ${ }^{37}$ also found out that treatment plan was altered in five patients $(17 \%)$ by PET/CT. The only study where PET/CT delineated larger volumes, $51.74 \mathrm{~cm}^{3}$, than CT, $46.26 \mathrm{~cm}^{3} \quad(p=0.043)$, measured the clinical target volume (CTV) which encompasses both GTV and an additional margin to cover for microscopic tumour spread. ${ }^{40}$

Comparisons of different PET/CT volume delineation methods demonstrated that auto-delineated (contoured) volumes were significantly smaller than subjective interpretation performed using visual inspection or manual delineation. ${ }^{35}$ Whereas, gradient-based (GR) auto-contouring method defined smaller TVs than signal-to-background ratio (SBR) auto-contouring method. ${ }^{34}$ In a study to compare six different functional volume delineation methods, Withofs et al. ${ }^{33}$ reported that all the delineated volumes were significantly different.

Closer inspection of Tables 2 and 3 shows that of the six papers on FDG-PET/CT compared with either MRI or CT, only two studies reported a change in treatment plan due to new lesions identified by FDG-PET/CT.

Five papers reported on IOV for TVs delineation (see Table 4). None of the studies on MRI sequences observed significant volume differences between observers and between modalities, indicating delineation similarities and consistencies for T2W, DWI and T2W-DWI, respectively. ${ }^{36,39}$ Two articles investigated IOV in GTV between PET/CT and CT and found significantly improved interobserver agreement with $\mathrm{PET} / \mathrm{CT}$ in comparison with $\mathrm{CT}$ $(p<0.001)$ and in addition, Buijsen et al. found the best interobserver agreement in auto-delineated PET/CT $(p<0 \cdot 001) .{ }^{35,37}$ Conversely, Withofs et al. $^{33}$ reported different interobserver variability between the various metabolic tumour volumes and GTV, and this was significant $(p<0.0001)$.

\section{Discussion}

This section provides a discussion on the findings of this review. The review demonstrates that TVs delineated on DWI-MR and FDG-PET/CT images are smaller compared to CT and T2W-MRI images. This implies that unnecessary inclusion of adjacent normal tissues in the planned TV was avoided. Further findings showed that PET/CT can aid adequate tumour coverage and can identify new lesions missed by MRI and CT.

Comparison of these findings with evidence from the previous review seems to be consistent only with data obtained from PET/CT and CT studies. ${ }^{29}$ It has been reported that a potential effect of PET/CT is either an increase or decrease in size of TVs. ${ }^{28}$ 
Existing guidelines such as the Radiation Therapy Oncology Group consensus guidelines ${ }^{42}$ on pelvic structures that may be included in a defined rectal TV should consist of the demonstrable tumour bed or GTV and the CTV which covers the GTV, mesorectum, pelvic lymph nodes, perirectal and presacral regions. It also includes the planning target volume (PTV) which covers the CTV, and an appropriate margin to account for variation in size, shape, position due to physiological mobility of structures in the pelvic region, and set-up error. ${ }^{19,42}$ The previous review ${ }^{29}$ and this current review identified few studies where PET/CT-TVs were significantly greater than CT-TVs. However, contrary to larger PET/CT-GTVs in the previous review, ${ }^{29}$ larger PET/CT-CTV values were reported in this current review. Several lines of evidence have established that PET/CT delineates smaller GTVs which practically approximate the true tumour. ${ }^{13,16,26,28,43}$ A possible explanation for the larger PET/CT-CTV result from this study is likely related to the inclusion of proximal and/or metastatic lymph nodes missed on CT in the CTV. ${ }^{28}$

Although both MRI findings are consistent with the literature, this present review found two studies that compared T2W-MRI with DW-MRI, whereas the earlier review reported three studies which compared MRI with CT and co-registered MRI-CT. The two studies in this review revealed that despite the differences in scanner quality and resolution, the standard T2W-MRI delineate larger TVs than DW-MRI-based approach. Even when compared with fused DW-T2W-MRI, DWI delineated the smallest volumes of the three approaches. A possible explanation for this might be based on the functional characteristics of DWI, that is, its ability to distinguish tumours from healthy tissues upon restricted diffusion which enhances tumour visibility. ${ }^{44,45}$

For studies that compared FDG-PET/CT and MRI, the results presented here have not previously been described. The findings showed that larger GTVs were defined with MRI than FDG-PET/ $\mathrm{CT}$; however, there are apparent potential for result bias as possible interferences of some of the imaging procedures adopted could not be ruled out. For example, in one of the studies rectal contrast was used to improve tumour conspicuity, ${ }^{34}$ whereas Regini et al. ${ }^{36}$ argued that this could have affected an overestimation of TV leading to larger volume delineated with MR. The observed larger volume with MRI could be attributed to the low resolution 1.5 T MR scanners used, no correction for geometric distortions and potential variations in the anatomical shape of the tumour due to physiological fillings in the rectum and bladder between both modalities. Furthermore, as revealed by Withofs et al., ${ }^{33}$ DICOM transfer of delineated TVs to the treatment planning system could probably have introduced added volume alterations.

On the IOV outcome, the results of this review showed interobserver delineation consistencies between T2W and DWI MRI sequences despite greater T2W volumes. However, a better interobserver agreement was found with PET/CT while PET/CT auto-delineation provided the best concordance compared to CT. These later findings are in line with those of previous review. An implication of this is that PET/CT and DW-MRI are not only reliable but also accurate for TVD. In contrast to earlier findings, however, observers with different levels of background were involved in the delineation process in the reports cited in this review (see Table 4). This finding may help us understand that both radiation oncologists and therapeutic radiographers should be able to take part in tumour delineation, so they can carry out their roles effectively and deliver high quality care. Experience acquired through training, peer-review and supervision can play an important role. ${ }^{35,39}$
Contrary to expectations, this review also highlights important issues, some of which are similar to those discussed in the previous review. First, the findings demonstrate a paucity of data since the last review. By contrast to previous review, specifically no published data comparing MRI and CT, and co-registered MRI and CT were identified, thereby restricting the review to limited comparisons. Furthermore, only two studies have attempted to investigate MRI sequences appropriate for rectal cancer delineation; two studies compared MRI with FDG-PET/CT while four studies have investigated PET/CT and CT. Second, gold standards, such as contouring method and imaging sequence, to validate and standardise TVD in PET/CT and MRI, respectively, are still lacking. Third, none of the included studies reported on rectal and bladder filling. Overestimation of CT volumes as observed in this review could possibly result from faeces in close proximity to the tumour. ${ }^{4,29}$ Finally, the use of FDG-PET/CT results in a change of treatment plan in about one out of six patients. ${ }^{37,38}$

This systematic review reveals that both DW-MR and PET/CT imaging can likely benefit rectal cancer patients. Although this finding to an extent is in consonance with the previous review and other evidence, it provides added advancement with functional DW-MRI as against the suggested standard T2W-MRI for rectal cancer treatment planning. ${ }^{13,29}$ An implication of this finding is the possibility that both functional imaging modalities can approximately define the boost volumes, that is, GTVs for dose escalation for local tumour control with minimal radiation-toxicity to OAR. However, in the context of this present era of advanced conformal radiotherapy, for example, IMRT, and coupled with the current shift from anatomical concept such as GTV, CTV and PTV to biological targeting concept, the combination of findings provide some support for the preliminary evidence that of the three modalities, PET/CT provides the best efficacy for image acquisition and TVD in rectal cancer radiotherapy planning. The biological target volume concept is fast becoming an important aspect in rectal cancer treatment as its major aims addresses boost volume, identifying new lesions as regard to nodal involvement and distant metastasis to avoid geographic misses, and dose painting, that is, heterogenous dose delivery to different regions of the tumour.

Another implication from these pooled findings is that the use of a sole imaging modality especially anatomical T2W-MR or CT imaging to delineate TVs may no longer be tenable in practice. However, with additional findings from PET/CT, it seems that multimodality which provides both metabolic and morphological information about the tumour as provided by PET/CT may be considered superior than single modality imaging in TVD. One of the included studies in this review ${ }^{34}$ co-registered FDG-PET and MRI GTVs but observed a mismatch of approximately $50 \%$ between both modalities. A few studies have been conducted with PET/ MRI modality in meningioma and head and neck tumours radiotherapy planning. ${ }^{46,47}$ However, despite this promising hybrid modality, questions remain and further studies are needed to determine how best to overcome the mismatch of the hybrid modality.

Furthermore, as it has been suggested, appropriate multimodality imaging (integrated or non-integrated) may be used for complementary purposes rather than substituting existing methods ${ }^{17,25,43}$ as absolute reliance on PET/CT may result in some geometrical misses especially in the evaluation of local tumour extent where MRI is indispensable. ${ }^{13,28}$ 


\section{Strengths and limitations}

The strengths of this review were the comprehensive and credible search strategy, acknowledgement of potential biases, inclusion of primary studies and use of the recommended QUADAS-2 quality assessment tool. However, there were also limitations as follows:

First, the quality of the included studies varied. Although there was no concern regarding the applicability of the studies; however, few of the studies were subjectively judged as 'at risk of bias' due to poor reporting and TVs not being delineated independently and blindly by observers. Second, majority of included studies were retrospective studies, often susceptible to unknown biases and hypothetical depiction of the results which might have impacted the reliability of the study conclusion. ${ }^{25}$ Third, nearly all the studies reviewed were single-centred with small sample sizes. This may have hampered the precision of the review. Fourth, none of the imaging modalities used in the reviewed studies were part of randomised controlled trials. A possible reason for this may be due to associated ethical challenges. ${ }^{28}$ Fifth, exclusion of non-English language publications may have resulted in missed studies which could have contributed to the outcome of the review. ${ }^{48}$ Sixth, five potentially eligible studies could not be retrieved. Finally, this review identified only eight studies since the last review in 2012. A limitation of this is possible threat to the generalisability of the findings. Notwithstanding, this study offers valuable insights into the amount of attention in the research literature that has been paid to imaging for TVD in rectal cancer despite the advances in imaging techniques and technology in external RTP.

\section{Conclusion}

This study evaluated the role of FDG-PET/CT and MRI in conjunction with conventional CT imaging in TVD in external radiotherapy planning of rectal cancers. This systematic review confirmed that FDG-PET/CT and DW-MRI delineated smaller TVs and showed better interobserver agreement compared to CT and T2W-MRI. FDG-PET/CT provided additional information by identifying new lesions missed by MRI and CT which resulted in a change of treatment plan for almost one in six patients with rectal cancer. The future of rectal cancer radiotherapy planning lies more with FDG-PET/CT and DW-MR imaging. The use of a single imaging modality, namely, CT, T2W-MR or PET-CT to delineate TV in rectal cancer radiotherapy planning is not acceptable; multimodality imaging should be advocated. Consequently, the findings from this systematic review do support strong recommendations of FDG-PET/CT and MRI combination for treatment planning of external radiation therapy for rectal cancer. Nevertheless, deciding on appropriate imaging modality for rectal cancer TVD should be a joint responsibility of a radiotherapy team or a designated body such as The European Society for Radiotherapy and Oncology and the IAEA.

\section{Implications for further research}

In total, 23 studies (previous $=15$ and current reviews $=8$ ) have been identified but the total number of patients so far enrolled $(n \leq 500)$ is too small to provide a basis for the generalisation of findings. Larger prospective multicentre studies, in particular, cross-sectional study designs as recommended by the Cochrane collaboration are needed to investigate these imaging modalities and patients' outcome, that is, treatment and survival outcome.
Supplementary material. To view supplementary material for this article, please visit https://doi.org/10.1017/S1460396921000388.

Acknowledgements. The authors are grateful to all the academic staff of the School of Health and Social Work, University of Hertfordshire, United Kingdom, who offered support and/or valuable comments on this work. Furthermore, the support of the library staff of the school in organising and executing the search strategy is appreciated.

This study was extracted from a thesis conducted in partial fulfilment for the award of a master's degree in Medical Imaging and Radiation Sciences at the University of Hertfordshire, United Kingdom. The first author would like to thank the Board of the University College Hospital, Ibadan, Nigeria, for granting the study leave to complete the master's degree programme.

Financial support. No financial support from any sources of funding

Conflicts of interest. The authors declare none.

\section{References}

1. Ferlay J, Soerjomataram I, Ervik M et al. GLOBOCAN 2012 v1.0, Cancer incidence and mortality worldwide: IARC cancerbase No. 11[Internet]. Lyon, France: International Agency for Research on Cancer, 2013. http:// globocan.iarc.fr. Accessed on $2^{\text {nd }}$ April 2018.

2. Bray F, Ferlay J, Soerjomataram I, Siegel RL, Torre LA, Jemal A. Global cancer statistics 2018: GLOBOCAN estimates of incidence and mortality worldwide for 36 cancers in 185 countries. CA Cancer J Clin 2018; 68: 394-424.

3. Siegel RL, Miller KD, Fedewa SA et al. Colorectal cancer statistics, 2017. CA Cancer J Clin 2017; 67: 177-193.

4. Tan J, Lim Joon D, Fitt G et al. The utility of multimodality imaging with CT and MRI in defining rectal tumour volumes for radiotherapy treatment planning: a pilot study. J Med Imaging Radiat Oncol 2010; 54 (6): 562-568.

5. McCarthy K, Pearson K, Fulton R, Hewitt J. Pre-operative chemoradiation for non-metastatic locally advanced rectal cancer. Cochrane Database Syst Rev 2012; (12): 1-28.

6. Brown KGM, Solomon, MJ, Mahon K, O'Shannassy S. Management of colorectal cancer. BMJ 2019; 366: 14561.

7. Rahbari NN, Elbers H, Askoxylakis V, Motschall E, Bork U, Büchler MW, et al. Neoadjuvant radiotherapy for rectal cancer: meta-analysis of randomized controlled trials. Ann of Surg Oncol 2013; 20: 4169-4182.

8. National Institute for Health and Care Excellence (NICE). Colorectal cancer: diagnosis and management. 2014. https://www.nice.org.uk/guidance/ cg131. Accessed on $3^{\text {rd }} 2014$ December.

9. O’Neill BD, Brown, G, Heald RJ, Cunningham D, Tait DM. Non-operative treatment after neoadjuvant chemoradiotherapy for rectal cancer. Lancet Oncol 2007; 8: 625-633.

10. Sauer R, Becker H, Hohenberger W, Rödel C, Wittekind C, Fietkau R, et al. Preoperative versus postoperative chemoradiotherapy for rectal cancer. N Engl J Med 2004; 351: 1731-1740.

11. Gérard J-P, Conroy T, Bonnetain F et al. Preoperative radiotherapy with or without concurrent fluorouracil and leucovorin in T3-4 rectal cancers: results of FFCD 9203. J Clin Oncol 2006; 24: 4620-4625.

12. Pereira GC, Traughber M, Muzic RF Jr. The role of imaging in radiation therapy planning: past, present, and future. Biomed Res Int 2014; 2014: 231090.

13. Wang YY, Zhe H. Clinical application of multimodality imaging in radiotherapy treatment planning for rectal cancer. Cancer Imaging 2013; 13: 495-501.

14. Appelt AL, Sebag-Montefiore, D. Technological advances in radiotherapy of rectal cancer: opportunities and challenges. Curr Opin Oncol 2016; 28 : 353-358.

15. Schlegel W. If you can't see it, you can miss it: the role of biomedical imaging in radiation oncology. Radiat Prot Dosim 2010; 139 (1-3): 321-326.

16. Buijsen J, van den Bogaard J, Janssen MH et al. FDG-PET provides the best correlation with the tumor specimen compared to MRI and CT in rectal cancer. Radiother Oncol 2011; 98: 270-276. 
17. Glide-Hurst CK, Low DA, Orton CG. MRI/CT is the future of radiotherapy treatment planning. Med Phys Point/Counterpoint 2014; 41: 110601.

18. Dirix P, Haustermans K, Vandecaveye V. The value of magnetic resonance imaging for radiotherapy planning. Semin Radiat Oncol 2014; 24 (3): 151-159.

19. Boldrini L, Placidi E, Dinapoli N et al. Hybrid Tri-Co-60 MRI radiotherapy for locally advanced rectal cancer: an in silico evaluation. tipsRO 2018; 6: 5-10.

20. Pollard JM, Wen Z, Sadagopan R, Wang J, Ibbott GS. The future of imageguided radiotherapy will be MR guided. Br J Radiol 2017; 90: 20160667.

21. Schmidt MA, Payne GS. Radiotherapy planning using MRI. Phys Med Biol 2015; 60: R323-R361.

22. Doemer A, Chetty IJ, Glide-Hurst C et al. Evaluating organ delineation, dose calculation and daily localization in an open-MRI simulation workflow for prostate cancer patients. Radiat Oncol 2015; 10: 37.

23. Wang H, Du K, Qu J, Chandarana H, Das IJ. Dosimetric evaluation of magnetic resonance-generated synthetic CT for radiation treatment of rectal cancer. PLoS One 2018; 13: e0190883.

24. Guerreiro F, Burgos N, Dunlop A et al. Evaluation of a multi-atlas CT synthesis approach for MRI-only radiotherapy treatment planning. Phys Med 2017; 35: 7-17.

25. Devic S. Towards biological target volumes definition for radiotherapy treatment planning: Quo Vadis PET/CT? J Nucl Med Radiat Ther 2013; 4 (3): 1-11.

26. Møller DS, Khalil AA, Knap MM, Muren LP, Hoffmann L. A planning study of radiotherapy dose escalation of PET-active tumour volumes in non-small cell lung cancer patients. Acta Oncol 2011; 50 (6): 883-888.

27. Scarsbrook AF, Barrington SF. PET-CT in the UK: current status and future directions. Clin Radiol 2016; 71: 673-690.

28. MacManus M, Nestle U, Rosenzweig KE et al. Use of PET and PET/CT for radiation therapy planning: IAEA expert report 2006-2007. Radiother Oncol 2009; 91: 85-94.

29. Gwynne S, Mukherjee S, Webster R et al. Imaging for target volume delineation in rectal cancer radiotherapy - a systematic review. Clin Oncol 2012; 24: 52-63.

30. Higgins JPT, Deeks JJ. Chapter 7: Selecting studies and collecting data. In: Higgins JPT, Green S (eds). Cochrane Handbook for Systematic Reviews of Interventions Version 5.1.0. The Cochrane Collaboration, 2011. http://www.handbook.cochrane.org. Accessed on March 2011.

31. Liberati A, Altman DG, Tetzlaff J et al. The PRISMA statement for reporting systematic reviews and meta-analyses of studies that evaluate healthcare interventions: explanation and elaboration. BMJ 2009; 339: b2700.

32. Whiting PF, Rutjes AW, Westwood ME et al. QUADAS-2: a revised tool for the quality assessment of diagnostic accuracy studies. Ann Intern Med 2011; 155: 529-536.

33. Withofs N, Bernard C, Van der Rest C et al. FDG PET/CT for rectal carcinoma radiotherapy treatment planning: comparison of functional volume delineation algorithms and clinical challenges. J App Clin Med Phys 2014; 15: 216-228.
34. Roels S, Slagmolen P, Nuyts J et al. Biological image-guided radiotherapy in rectal cancer: challenges and pitfalls. Int J Radiat Oncol Biol Phys 2009; 75: 782-790.

35. Buijsen J, van den Bogaard J, van der Weide H et al. FDG-PET-CT reduces the interobserver variability in rectal tumor delineation. Radiother Oncol 2012; 102: 371-376.

36. Regini F, Gourtsoyianni S, Cardoso De Melo R et al. Rectal tumour volume (GTV) delineation using T2-weighted and diffusion-weighted MRI: implications for radiotherapy planning. Eur J Radiol 2014; 83: 768-772.

37. Whaley JT, Fernandes AT, Sackmann R et al. Clinical utility of integrated positron emission tomography/computed tomography imaging in the clinical management and radiation treatment planning of locally advanced rectal cancer. pro 2014; 4: 226-232.

38. Braendengen $M$, Hansson $K$, Radu C, Siegbahn A, Jacobsson H, Glimelius B. Delineation of gross tumor volume (GTV) for radiation treatment planning of locally advanced rectal cancer using information from MRI or FDG-PET/CT: a prospective study. Int J Radiat Oncol Biol Phys 2011; 81: 439-445.

39. Burbach JPM, Kleijnen JPJ, Reerink $O$ et al. Inter-observer agreement of MRI-based tumor delineation for preoperative radiotherapy boost in locally advanced rectal cancer. Radiother Oncol 2016; 118: 399-407.

40. Kilic D, Catli S, Ulger S, Kapucu LO. Is there any impact of PET/CT on radiotherapy planning in rectal cancer patients undergoing preoperative IMRT? Turk J Med Sci 2015; 45: 129-135.

41. Janssen MH, Aerts HJ, Öllers MC et al. Tumor delineation based on timeactivity curve differences assessed with dynamic fluorodeoxyglucose positron emission tomography-computed tomography in rectal cancer patients. Int J Radiat Oncol Biol Phys 2009; 73: 456-465.

42. Gay HA, Barthold HJ, O'Meara E et al. Pelvic normal tissue contouring guidelines for radiation therapy: a radiation therapy oncology group consensus panel atlas, Int J Radiat Oncol Biol Phy 2012; 83: e353-e362.

43. Bhatnagar P, Subesinghe M, Patel C, Prestwich R, Scarsbrook AF. Functional imaging for radiation treatment planning, response assessment, and adaptive therapy in head and neck cancer. Radiographics 2013; 33: 1909-1929.

44. Kaur H, Choi H, You YN et al. MR imaging for preoperative evaluation of primary rectal cancer: practical considerations. Radiographics 2012; 32 (2): 389-409.

45. Nguyen TLF, Soyer P, Fornès P, Rousset P, Kianmanesh R, Hoeffel C. Diffusion-weighted MR imaging of the rectum: clinical applications. Crit Rev Oncol 2014; 92: 279-295.

46. Thorwarth D, Henke G, Muller A-C et al. Simultaneous 68Ga-DOTATOCPET/MRI for IMRT treatment planning for meningioma: first experience. Int J Radiat Oncol Biol Phys 2011; 81: 273-283.

47. Thorwarth D, Leibfarth S, Mönnich D. Potential role of PET/MRI in radiotherapy treatment planning. Clin Transl Imaging 2013; 1: 45-51.

48. Coughlan M, Cronin P. Doing a Literature Review in Nursing, Health and Social Care, 2nd edition. London: Sage Publication Ltd, 2017. 